\title{
Bitter taste receptor T2R1 activities were compatible with behavioral sensitivity to bitterness in chickens
}

\author{
Nozomi Hirose $^{\mathrm{a}, 1}$, Yuko Kawabata ${ }^{\mathrm{b}, 1}$, Fuminori Kawabata ${ }^{\mathrm{c},}{ }^{\text {, }}$ \\ Shotaro Nishimura ${ }^{\text {a,d }}$, and Shoji Tabata ${ }^{\text {a,d }}$
}

a Laboratory of Functional Anatomy, Department of Bioresource Sciences, Graduate School of Bioresource and Bioenvironmental Sciences, Kyushu University, Fukuoka, Japan

${ }^{\mathrm{b}} \mathrm{School}$ of Agriculture, Kyushu University, Fukuoka, Japan

'Institute for Advanced Study, Kyushu University, Fukuoka, Japan

${ }^{\mathrm{d}}$ Faculty of Agriculture, Kyushu University, Fukuoka, Japan

*Corresponding author: Fuminori Kawabata, Institute for Advanced Study, Kyushu University, 6-10-1 Hakozaki, Higashi-ku, Fukuoka 812-8581, Japan. Tel.: +81-92-642-2944. Fax: +81-92-642-2944

E-mail address: kawabata@agr.kyushu-u.ac.jp

${ }^{1}$ These authors contributed equally to this work. 


\section{ABSTRACT}

Clarification of the mechanism of the sense of taste in chickens will provide information useful for creating and improving new feedstuffs for chickens, because the character of the taste receptors in oral tissues affects feeding behavior in animals. In this study, we focused on the sensitivity to bitterness in chickens. We cloned one of the bitter taste receptors, $T 2 R 1$, from the chicken palate, constructed several biosensor-cells expressing chicken T2R1 (cT2R1), and determined a highly sensitive biosensor of cT2R1 among them. By using $\mathrm{Ca}^{2+}$ imaging methods, we identified two agonists of cT2R1, dextromethorphan (Dex) and diphenidol (Dip). Dex was a new agonist of cT2R1 that was more potent than Dip. In a behavioral drinking study, the intake volumes of solutions of these compounds were significantly lower than that of water in chickens. These aversive concentrations were identical to the concentrations that could activate cT2R1 in a cell-based assay. These results suggest that the cT2R1 activities induced by these agonists are linked to behavioral sensitivity to bitterness in chickens.

Key words: bitter, T2R1, taste receptor, chicken 


\section{Introduction}

In chickens, the sense of taste is one of the most important senses for acquiring and

choosing feeds, as are the senses of smell and vision [1]. The elucidation of the sense of taste in chickens offers opportunities to create and improve feedstuffs for chickens and to contribute to the understanding of mammalian senses of taste in comparison with the functioning of mammalian taste receptors. In chickens, there are four taste receptor genes (for bitter, umami, sour, and salty) among the five basic tastes recognized by humans; the $T 1 R 2$ gene, which is the sweet taste receptor gene, is absent [1]. Recently, a number of articles about chicken taste receptors have been published. Behrens et al. reported that the chicken bitter taste receptors, T2R1, T2R2, and T2R7, are broadly activated by many bitter substances [2]. Baldwin et al. reported that the chicken umami receptor, T1R1/T1R3, is activated by L-alanine and L-serine [3]. Furthermore, we elucidated that the chicken fat taste receptor, GPR120, in the oral cavity recognizes oleic acid and linoleic acid [4]. However, there are many open questions concerning the taste transduction system in chickens compared with those in mammals.

In this study, we focused on the sensitivity to bitterness in chickens. To supply sufficient chicken products in the future, it is necessary to develop new feedstuffs from by-products of food-processing and food residues [5]. Because many by-products of food-processing and food 
residues are derived from plants, it is believed that many bitter compounds are contained in these materials. Thus, elucidating the chicken taste system with respect to bitterness is important in the development of new feedstuffs for chickens.

Although some agonists of chicken T2R1 (cT2R1), T2R2 (cT2R2), and T2R7 (cT2R7), were identified [2], it is not clear whether there is a strong connection between the activity of bitter taste receptors and behavioral sensitivity to bitterness in chickens. In this study, we cloned the $c T 2 R 1$ gene from the palate, constructed several biosensor cells expressing cT2R1, and determined a highly sensitive biosensor of cT2R1 among them. We found a new agonist of cT2R1 by using the biosensor, and found that the activities of cT2R1 agonists are linked to aversive behavior in chickens.

\section{Materials and methods}

\subsection{Chemicals}

Dextromethorphan (Dex) hydrochloride, thiamine (Thi) hydrochloride, and adenosine

triphosphate (ATP) were purchased from Sigma-Aldrich (St. Louis, MO, USA). Diphenidol (Dip) hydrochloride was purchased from Wako Pure Chemical Industries (Osaka, Japan). These compounds were dissolved in ultrapure water or ethanol and stored at $-20^{\circ} \mathrm{C}$ as stock solutions. 
These stock solutions were diluted with a standard bath solution containing $140 \mathrm{mM} \mathrm{NaCl}, 5$ $\mathrm{mM} \mathrm{KCl}, 2 \mathrm{mM} \mathrm{MgCl}, 2 \mathrm{mM} \mathrm{CaCl}, 10 \mathrm{mM}$ HEPES and $10 \mathrm{mM}$ glucose at $\mathrm{pH} 7.4$, adjusted with $\mathrm{NaOH}$ just before each experiment.

\subsection{Animals}

Rhode Island Red strain 0- to 1-wk-old chicks were used for this research. The study was carried out according to the Guide for Animal Experiments issued by Kyushu University, the Law Concerning the Human Care and Control of Animals (Law No. 105; October 1, 1973), and the Japanese Government Notification on the Feeding and Safekeeping of Animals (Notification No. 6; March 27, 1980).

\subsection{Constructions of chicken T2R1, chicken gustducin, and $G \alpha_{16 / g u s t 44}$}

Total RNA was isolated from the palates of chicks, and first-strand cDNA was synthesized by reverse transcription. Then, deduced open reading frames (ORFs) of $c T 2 R 1$ and chicken gustducin (c-gust) were amplified and sequenced. The polymerase chain reaction (PCR) primers were designed based on the genome assemblies of the cT2Rl (AB249766.1) and c-gust (NM_001267811.1). The PCR products of the ORFs were subcloned into the pDisplay (Life 
Technologies Japan, Tokyo), pCI (Promega KK, Tokyo), or pcDNA3.1(+) (Life Technologies Japan) expression vectors by using the In-Fusion HD Cloning Kit (Takara Bio, Otsu, Japan). G $\alpha_{16 / g u s t 44} /$ pcDNA3.1(+) was kindly donated by Dr. Takashi Ueda (Nagoya City University) [6]. The entire sequences of $c T 2 R 1, c$-gust, and $G \alpha_{16 / g u s t 44}$ were confirmed.

\subsection{Cell culture}

Human embryonic kidney (HEK)-derived 293T (HEK293T) cells were maintained in

Dulbecco's modified Eagle's medium (DMEM high glucose, Wako) containing 10\% fetal bovine serum (FBS, GE Healthcare, Buckinghamshire, UK), and penicillin-streptomycin solution $(\times 100)\left(\right.$ Wako) at $37^{\circ} \mathrm{C}$ in $5 \% \mathrm{CO}_{2}$.

\section{5. $\mathrm{Ca}^{2+}$ imaging by confocal microscopy}

For the $\mathrm{Ca}^{2+}$ imaging experiments, HEK293T cells were transfected with either empty

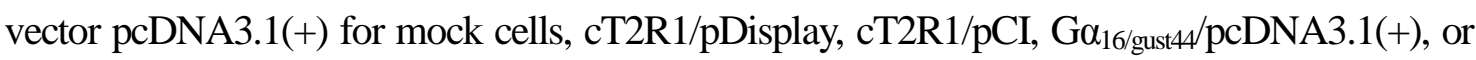
c-gust/pcDNA3.1(+) by using ScreenFect ${ }^{\mathrm{TM}}$ A (Wako) on coverslips coated with poly-D-lysine $(0.1 \mathrm{mg} / \mathrm{mL})$. After transfection, the cells were incubated for $48 \mathrm{~h}$ at $37^{\circ} \mathrm{C}$ and $5 \% \mathrm{CO}_{2}$. Then, the cells were loaded with $1.25 \mu \mathrm{M}$ Fluo 4 -AM solution for $30 \mathrm{~min}$ at $37^{\circ} \mathrm{C}$ and $5 \% \mathrm{CO}_{2}$ in the 
dark. Fluo 4-AM solution was prepared according to the manufacturer's manual (Dojindo Laboratories, Kumamoto, Japan).

The cover slips were washed with the standard bath solution, and Fluo-4 fluorescence was measured in the standard bath solution using a confocal laser scanning microscope (Nikon A1R, Nikon Co., Tokyo). The coverslips were mounted in a chamber connected to a gravity flow system to deliver various stimuli. Chemical stimulation was applied by running a bath solution containing various chemical reagents. Cell viability was confirmed by responses to $3 \mu \mathrm{M}$ ATP. The ATP activity was also used for normalizing cT2R1 activity in each experiment.

\subsection{The one-bowl drinking test}

Rhode Island Red strain chicks were raised in a box brooder (Showa Furanki, Saitama, Japan) under $24 \mathrm{~h}$ lighting. Tap water was used as the chicks' drinking water. The experiment was done at about $20-30^{\circ} \mathrm{C}$ in the same box brooder that was familiar to the chicks. The chicks were kept in individual spaces divided by woven metal wire, reducing the stress created by seeing other chicks. Commercial feedstuff was freely fed to the chicks during this experiment (Powerlayer17Y, JA Kitakyushu Kumiai Shiryo, Fukuoka, Japan). On day 1, the chicks were given tap water freely. In the training period (days 2-6), the chicks were given water for only 10 
min total in each 24-h period beginning at 17:00 to train them in drinking for a short time only.

In the experiment period (days 7 and 8), the chicks were given water or a bitter solution for 10 min beginning at 17:00 as in the training period. The order in which water and the bitter solution were given was randomized over the two days. To compensate for evaporation from the bowl in the 10 min of exposure, control tap water was set in the box brooder, and the amount of evaporation was subtracted from the volume of water drunk. Bitter stock solutions were diluted with tap water in the drinking test.

\subsection{Statistical analysis}

The data are expressed as means \pm SEM. Statistical analyses were done using the paired $t$-test, unpaired $t$-test, or Tukey-Kramer test. The analyses, calculations of $\mathrm{EC}_{50}$ values, and illustrations of fitting curves using the Hill equation were performed using the IGOR Pro software package (Version 6.34J, WaveMetrics, Portland, OR), and differences with $p$-values $<0.05$ were considered significant.

\section{Results}

\subsection{Cloning of chicken T2R1 and gustducin from palate}


Since mRNAs of $c T 2 R 1$ and $c$-gust have been detected in the chicken palate by RT-PCR

[7,8], we cloned the $c T 2 R 1$ and $c$-gust genes from a chicken palate. After amplification in E. coli, we confirmed that the cDNA sequences of the cloned $c T 2 R I$ and $c$-gust matched those in the genomic sequences of the NCBI database (AB249766.1 and NM_001267811.1, respectively) except for some bases (Supplement Figs. 1, 2). The translated product of the cloned $c T 2 R 1$ and c-gust genes matched the database (AB249766.1 and NM_001267811.1, respectively) with the exception of two amino acids.

\subsection{Determination of functional bitter biosensor in chickens using cT2Rl-expressing cells}

We made three types of cT2R1-expressing cells; cells transfected with the following: 1)

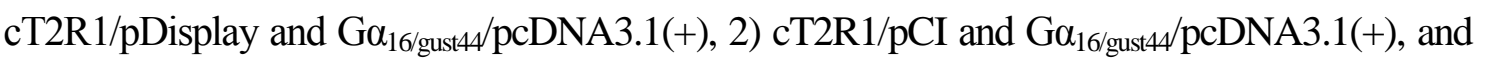
3) cT2R1/pDisplay and c-gust/pcDNA3.1(+). Because Dip was reported to be an agonist of cT2R1 [2], we screened a highly sensitive bitter taste biosensor among three types cells by using

Dip. First, in the cT2R1/pDisplay-c-gust cells, the increase of the intracellular $\mathrm{Ca}^{2+}$ concentration by Dip was significantly smaller than that in the cT2R1/pDisplay-G $\alpha_{16 / \text { gust } 44}$ cells (Fig. 1A).

Second, in the cT2R1/pCI-G $\alpha_{16 / g u s t 44}$ cells, the increase of the intracellular $\mathrm{Ca}^{2+}$ concentration by Dip was also significantly smaller than that in the cT2R1/pDisplay-G $\alpha_{16 / g u s t 44}$ cells (Fig. 1B). 
These results suggested that the expression of $\mathrm{G} \alpha_{16 / g u s t} 44$ and the use of the pDisplay vector were the most suitable for constructing the cT2R1-expressing bitter biosensor among the variations tested. We also confirmed the availability of the cT2R1/pDisplay-G $\alpha_{16 / g u s t 44}$ cells using Dex (Fig. 1A, B), the new agonist of cT2R1 described below.

3.3. Dextromethorphan and diphenidol increased cytosolic $\mathrm{Ca}^{2+}$ concentrations through cT2RI

By $\mathrm{Ca}^{2+}$ imaging in cT2R1/pDisplay-G $\alpha_{16 / g u s t 44}$ cells, we assayed three bitter substances in human T2Rs agonists [9]. After exposure to the stimuli of $10 \mu \mathrm{M}$ Dex and $100 \mu \mathrm{M}$ Dip, the cytosolic $\mathrm{Ca}^{2+}$ concentration was increased (Fig. 2A). On the other hand, the stimulus of $100 \mu \mathrm{M}$ Thi did not change the $\mathrm{Ca}^{2+}$ concentration (Fig. 2A). The mock cells were not affected by Dex or Dip (Fig. 2B). Representative images of the $\mathrm{Ca}^{2+}$ concentration changes induced by Dex and Dip are shown in Fig. 2C. There were significant differences in the cytosolic $\mathrm{Ca}^{2+}$ concentration between the cT2R1/pDisplay-G $\alpha_{16 / g u s t} 44$ cells and the mock cells with both Dex and Dip (Fig. 2D). After two agonist candidates were found, we examined whether these compounds activated cT2R1 dose-dependently. These two compounds showed dose-dependency in cT2R1/pDisplay-G $\alpha_{16 / g u s t 44}$ cells (Figs. 3C, D). Dex was exposed to new cT2R1 agonists, and the cT2R1 activities of Dip and Thi were identical to those detailed in the previous report [2]. 
The $\mathrm{EC}_{50}$ values of Dex and Dip for cT2R1 were $2.81 \mu \mathrm{M}$ and $65.7 \mu \mathrm{M}$, respectively. The cT2R1 activity of Dex was stronger than that of Dip.

\subsection{One-bowl drinking tests of bitter solutions in chickens}

Because Dex and Dip were identified as agonists of cT2R1, we then examined whether chickens show aversive behavior in response to these compounds. We determined three doses of bitter solutions by reference to the results shown in Figs. 3C and D. The lowest dose was set to the initial rise concentration of cT2R1 activity. The middle and highest doses were set to the near plateau and full plateau concentrations, respectively. In the one-bowl drinking test, the intake volumes of $10 \mu \mathrm{M}$ and $100 \mu \mathrm{M}$ Dex solutions per body weight for 10 min were significantly smaller than that of water per body weight (Fig. 3A). The value of $1 \mu \mathrm{M}$ Dex solution was not different from that of water. The intake volumes of $300 \mu \mathrm{M}$ and $5 \mathrm{mM}$ Dip solutions per body weight were significantly smaller than that of water per body weight (Fig. 3B). No differences between the $30 \mu \mathrm{M}$ Dip solution and water were observed.

\section{Discussion}

In this study, we identified a new cT2R1 agonist, Dex. Chickens drank less Dex solution 
than water over the course of $10 \mathrm{~min}$ in a drinking test. Because 10 min was not long enough for the solution to be absorbed in the gastrointestinal tract, it is thought that chickens can detect Dex in the oral tissues via cT2R1. Furthermore, we found that the dose-dependency of the cT2R1 activity induced by Dex and Dip was linked to drinking behavior in chickens. These results suggest that the cT2R1 activity induced by bitter compounds is compatible with the behavioral sensitivity to bitterness in chickens, and that the present constructed bitter taste biosensor is a good index for predicting the sensitivity to bitterness in chickens.

In the present study, we constructed three types of cT2R1-expressing cells and confirmed that the pDisply vector and $\mathrm{G} \alpha_{16 / g u s t 44}$ are suitable for constructing a highly sensitive bitter taste biosensor for chickens. It is presumed that the efficacy of the expression of cT2R1 on the cell membrane was increased by these materials. It is known that T2Rs are difficult to express on cell membranes because their extracellular region is too short [10]. Since the pDisplay vector is designed to anchor the target protein expressed from this vector to the cell membrane and to display it on the extracellular side, this vector might increase the expression efficacy on the cell membrane in comparison with the expression efficacy obtained using the pCI vector. On the other hand, $\mathrm{G \alpha}_{16 / \text { gust } 44}$ is broadly used to assay the activity of various taste receptors [6]. Thirty-seven C-terminal amino acids of $\mathrm{G}_{16 / \text { gust } 44}$ coupled with $\mathrm{T} 2 \mathrm{Rs}$, and the $37 \mathrm{C}$-terminal 
amino acids of chicken gustducin cloned in this study were almost identical to $37 \mathrm{C}$-terminal amino acids of $\mathrm{G} \alpha_{16 / \text { gust } 44}$ with the exception of two amino acids (Supplement Fig. 2). Because the sensitivity of cT2R1- and c-gust-expressing cells was significantly lower than that of cT2R1and $\mathrm{G} \alpha_{16 / g u s t 44}$-expressing cells, the $\mathrm{G} \alpha_{16}$ part of $\mathrm{G} \alpha_{16 / \text { gust } 44}$ is thought to be important in the construction of a sensitive biosensor of cT2R1.

In this study, we found that Dex is a new cT2R1 agonist. Because the cT2R1 activity induced by Dex was stronger than that induced by Dip, Dex is assumed to be one of the leading compounds in the study of cT2R1. Dex activates only human T2R1 and T2R10 among 25 human T2Rs [9]. Because, as Behrens et al. reported, chicken T2Rs are broadly tuned receptors for detecting various bitter compounds [2], cT2R2 and cT2R7 may be activated by Dex. In the present drinking test, the intake volume of $5 \mathrm{mM}$ Dip solution was about $50 \%$ of the water intake volume. On the other hand, the intake volume of $100 \mu \mathrm{M}$ Dex was about $85 \%$ of the water intake volume. These results implied that chickens may have a stronger aversion to Dip than to Dex. Since the cT2R1 activities of $100 \mu \mathrm{M}$ Dex and $5 \mathrm{mM}$ Dip were almost the same, the differences in the other cT2Rs activities of Dex and Dip may induce the differences in aversive behaviors observed in this study. Further studies are needed to elucidate whether Dex activates cT2R2 and cT2R7. 
In summary, the present study revealed that the cT2R1 activities induced by Dex and Dip were compatible with the behavioral sensitivity to bitterness in chickens. The assay system constructed in this study will be a useful tool in developing new feedstuffs for chickens and comparing chicken taste receptors with mammalian taste receptors in the study of animal taste systems.

\section{Acknowledgments}

We thank Dr Takashi Ueda (Nagoya City University) for providing the $\mathrm{G} \alpha_{16 / g u s t 44}$ plasmid. We appreciate the technical assistance of The Research Support Center, Research Center for Human Disease Modeling, Kyushu University Graduate School of Medical Sciences. This study was supported in part by a grant to F.K. from the Funds for the Development of Human Resources in Science and Technology, the Japan Science and Technology Agency. This work was also supported by a grant to F.K. from JSPS KAKENHI (\# 26850207). 


\section{References}

[1] E. Roura, M.W. Baldwin, K.C. Klasing, The avian taste system: Potential implications in poultry nutrition, Anim. Feed Sci. Technol. 180 (2013) 1-9.

[2] M. Behrens, S.I. Korsching, W. Meyerhof, Tuning properties of avian and frog bitter taste receptors dynamically fit gene repertoire sizes, Mol. Biol. Evol. 31 (2014) 3216-3227.

[3] M.W. Baldwin, Y. Toda, T. Nakagita, M.J. O'Connell, K.C. Klasing, T. Misaka, S.V. Edwards, S.D. Liberles, Evolution of sweet taste perception in hummingbirds by transformation of the ancestral umami receptor, Science 345 (2014) 929-933.

[4] R. Sawamura, Y. Kawabata, F. Kawabata, S. Nishimura, S. Tabata, The role of G-protein-coupled receptor 120 in fatty acids sensing in chicken oral tissues, Biochem. Biophys. Res. Commun. doi:10.1016/j.bbrc.2015.01.125 (2015).

[5] M.J. Boland, A.N. Rae, J.M. Vereijken, M.P.M. Meuwissen, A.R.H. Fischer, M.A.J.S. van Boekel, S.M. Rutherfurd, H. Gruppen, P.J. Moughan, W.H. Hendriks, The future supply of animal-derived protein for human consumption, Trends Food Sci. Technol. 29 (2013) 62-73.

[6] T. Ueda, S. Ugawa, H. Yamamura, Y. Imaizumi, S. Shimada, Functional interaction between T2R taste receptors and G-protein alpha subunits expressed in taste receptor cells, J. Neurosci. 23 (2003) 7376-7380.

[7] K. Kudo, K. Wakamatsu, S. Nishimura, S. Tabata, Gustducin is expressed in the taste buds of the chicken, Anim. Sci. J. 81 (2010) 666-672.

[8] S.L. Cheled-Shoval, M. Behrens, W. Meyerhof, M.Y. Niv, Z. Uni, Perinatal administration of a bitter tastant influences gene expression in chicken palate and duodenum, J. Agric. Food Chem. 62 (2014) 12512-12520.

[9] W. Meyerhof, C. Batram, C. Kuhn, A. Brockhoff, E. Chudoba, B. Bufe, G. Appendino, M. Behrens, The molecular receptive ranges of human TAS2R bitter taste receptors, Chem. Senses 35 (2010) 157-170.

[10] J. Chandrashekar, K.L. Mueller, M.A. Hoon, E. Adler, L. Feng, W. Guo, C.S. Zuker, N.J. Ryba, T2Rs function as bitter taste receptors, Cell 100 (2000) 703-711. 


\section{Figure legends}

Fig. 1. Availability of three types of cT2R1-expressing biosensor cells analyzed by $\mathrm{Ca}^{2+}$ imaging. (A) The increases in the $\mathrm{Ca}^{2+}$ concentration induced by $10 \mu \mathrm{M}$ dextromethorphan (Dex) and $100 \mu \mathrm{M}$ diphenidol (Dip) stimuli in the cells transfected with cT2R1/pDisplay and $\mathrm{G} \alpha_{16 / \text { gust } 4 / \mathrm{pcDNA} 3.1(+)}$ were significantly larger than those in the cells transfected with cT2R1/pDisplay and c-gust/pcDNA3.1(+). (B) The increases in the $\mathrm{Ca}^{2+}$ concentration induced by $10 \mu \mathrm{M}$ Dex and $100 \mu \mathrm{M}$ Dip stimuli in the cells transfected with cT2R1/pDisplay and Ga $\alpha_{16 / g u s t 44} / \mathrm{pcDNA} 3.1(+)$ were significantly larger than those in the cells transfected with

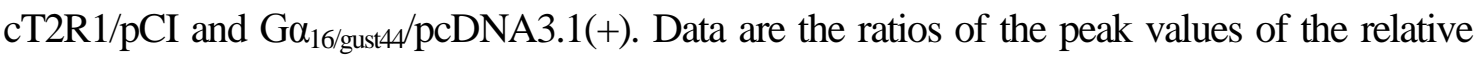
fluorescence units after $10 \mu \mathrm{M}$ Dex or $100 \mu \mathrm{M}$ Dip stimuli following $3 \mu \mathrm{M}$ ATP stimulus. The data are means \pm SEM of $111-266$ cells per bar. ${ }^{*} p<0.01$, and ${ }^{* * *} p<0.0001$ by unpaired $t$-test.

Fig. 2. Dextromethorphan (Dex) and diphenidol (Dip) increased cytosolic $\mathrm{Ca}^{2+}$ concentrations through cT2R1. (A) The representative data of the cytosolic $\mathrm{Ca}^{2+}$ increase induced by $10 \mu \mathrm{M}$ Dex and $100 \mu \mathrm{M}$ Dip stimuli in the cells transfected with cT2R1/pDisplay and Go $\alpha_{16 / \text { gust } 4 / \mathrm{pcDNA3}}$.1(+). The stimulus with $100 \mu \mathrm{M}$ thiamine (Thi) did not change the $\mathrm{Ca}^{2+}$ concentration. Bars indicate the duration of the chemical applications. Data are the ratios of the 
relative fluorescence units (RFU) to the baseline value. ATP was applied to confirm the cell viability. (B) Three bitter compounds did not increase the cytosolic $\mathrm{Ca}^{2+}$ concentration in the cells transfected with empty vector (Mock). (C) Changes in cytosolic $\mathrm{Ca}^{2+}$ concentrations after bitter substance stimuli, as indicated by the Fluo-4 ratio with pseudo-color expression. (D) Dex and Dip significantly increased the cytosolic $\mathrm{Ca}^{2+}$ concentration in cT2R1-expressing cells compared with mock cells. Data are the ratio of the RFU peak value after Dex or Dip stimulus to $3 \mu \mathrm{M}$ ATP. Data are the means \pm SEM of 5 coverslips' data. One coverslip had approx. 100 cells, and the averages of the data from the 100 cells were treated as one coverslip's data. ${ }^{* * *} p<0.0001$ by unpaired $t$-test.

Fig. 3. The relationships between aversive behavior and cT2R1 activity in chickens. (A) Solution intakes per body weight over $10 \mathrm{~min}$ in the one-bowl drinking test in chickens. The intakes of $10 \mu \mathrm{M}$ and $100 \mu \mathrm{M}$ dextromethorphan (Dex) solutions were significantly smaller than the water intake. On the other hand, the value for $1 \mu \mathrm{M}$ Dex solution was not different from that for water. This dose dependency was matched to the cT2R1 activity of Dex described below figure (C). Data are the means $\pm \operatorname{SEM}(\mathrm{n}=5-7) .{ }^{* *} p<0.01$ by paired $t$-test. (B) The intakes of 300 $\mu \mathrm{M}$ and $5 \mathrm{mM}$ diphenidol (Dip) solutions were significantly smaller than the water intake. The 
value of the $30 \mu \mathrm{M}$ Dip solution was not different from that of water. This dose dependency was matched to the cT2R1 activity of Dip described below figure (D) as well as to that of Dex. Data are the means \pm SEM $(\mathrm{n}=5-7) . \stackrel{*}{p}<0.05$, and ${ }^{* *} p<0.01$ by paired $t$-test. $(\mathrm{C}, \mathrm{D})$ The dose-dependency of cT2R1 activation by Dex and Dip in the cells transfected with

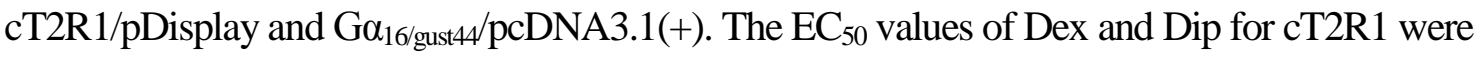
$2.81 \mu \mathrm{M}$ and $65.7 \mu \mathrm{M}$, respectively. Data are the ratios of the RFU peak values after each dose of Dex or Dip stimulus to $3 \mu \mathrm{M}$ ATP. Data are the means \pm SEM of 3-5 coverslips' data. One coverslip had approx. 50-100 cells, and the averages of the data for the 50-100 cells were treated as one coverslip's data. Arrowed lines show the relationships between each dose used in the behavioral study described above (A, B) and the dose-dependency curve of cT2R1 with these compounds. 


\section{Figures}
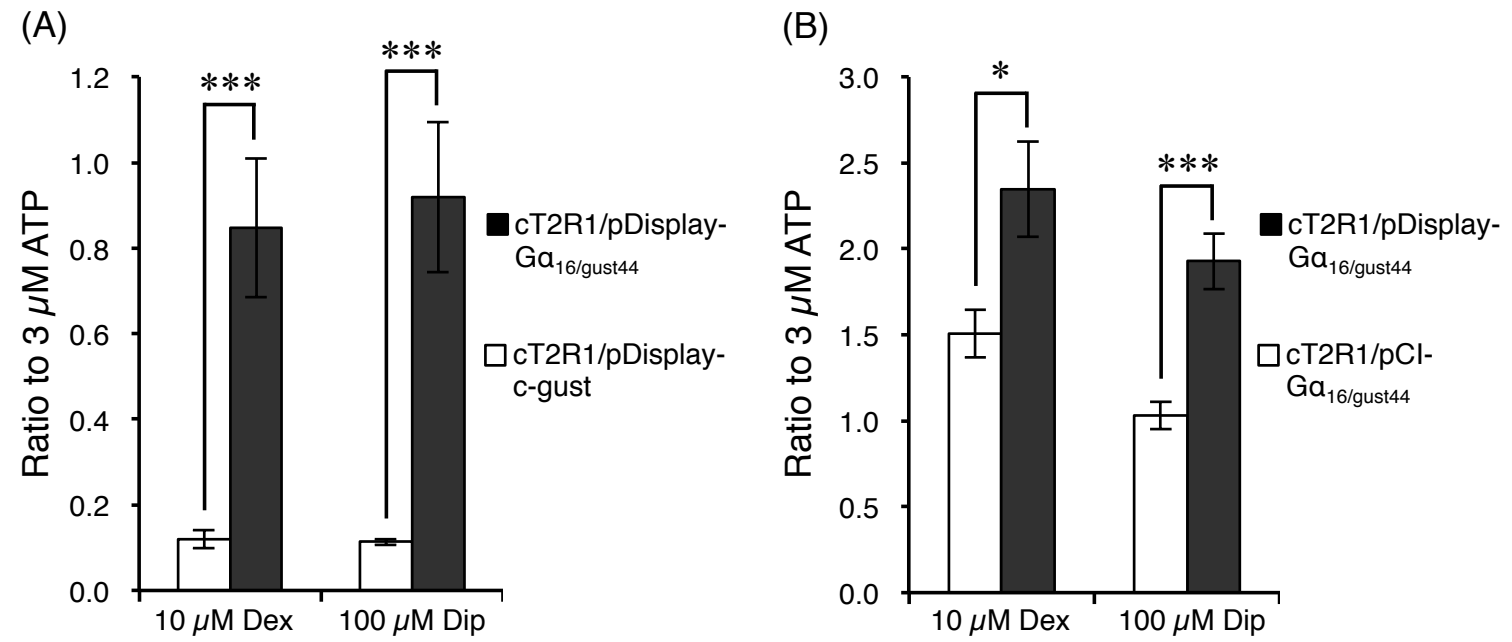

Figure 1

Hirose et al. 


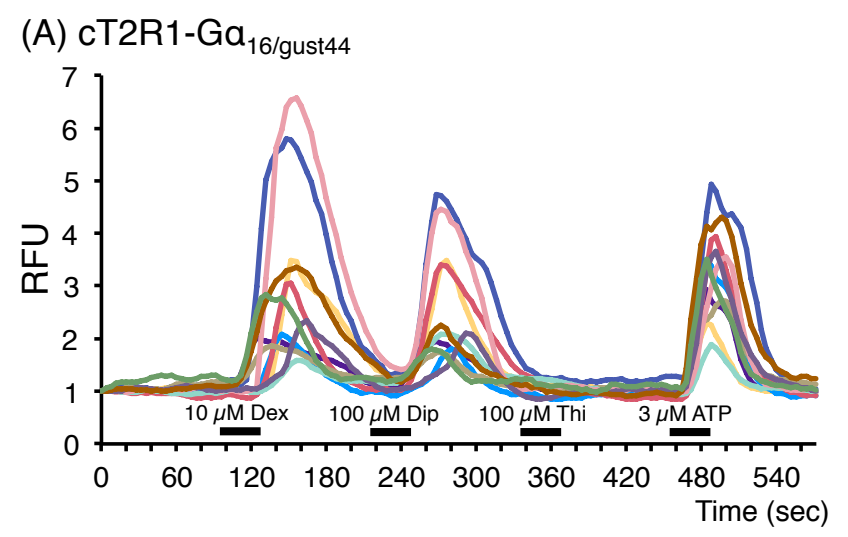

(C)

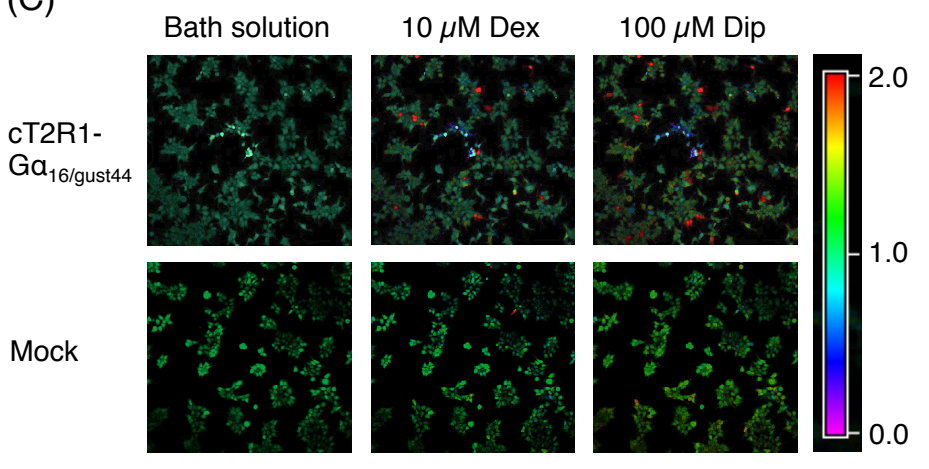

(B) Mock

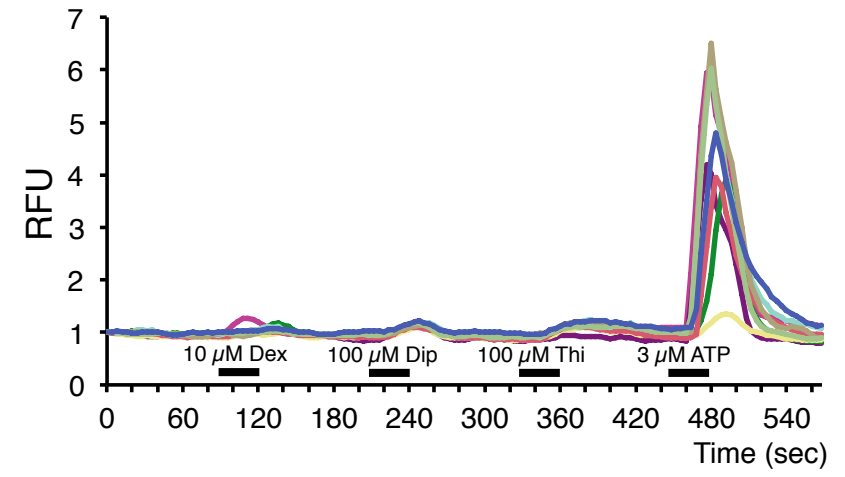

(D)

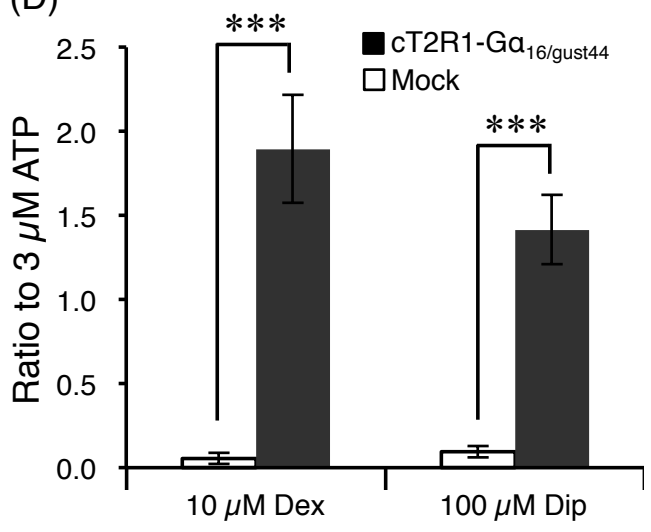

Figure 2

Hirose et al. 
(A)

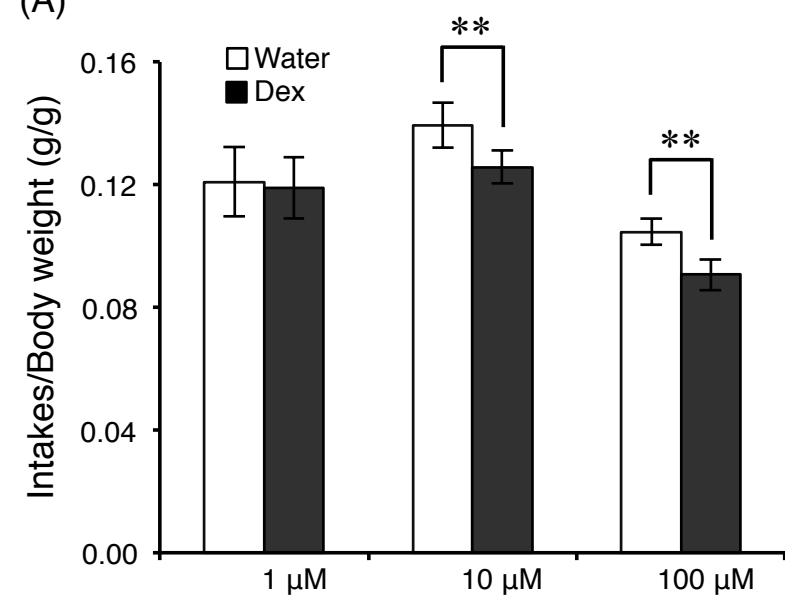

(C)

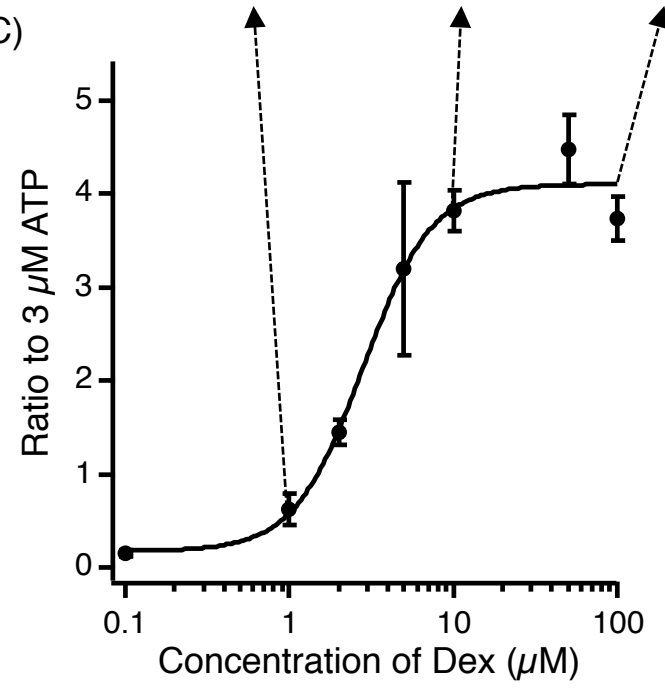

(B)

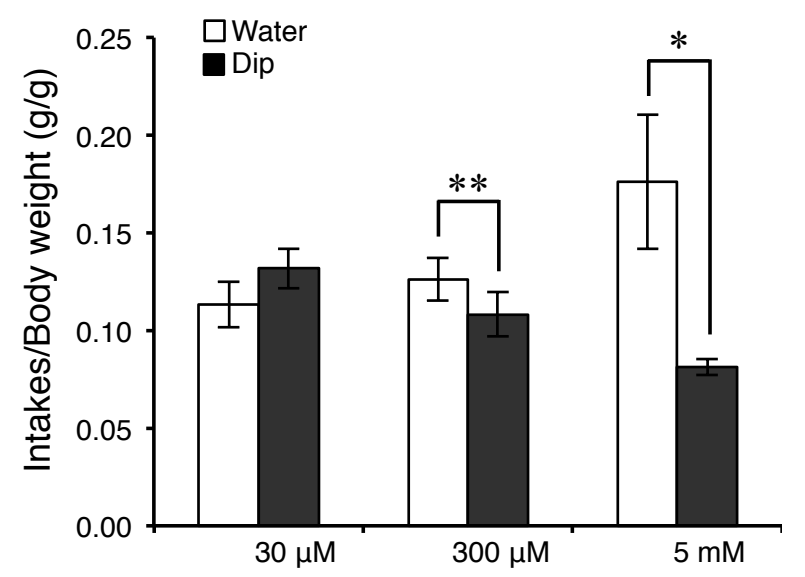

(D)

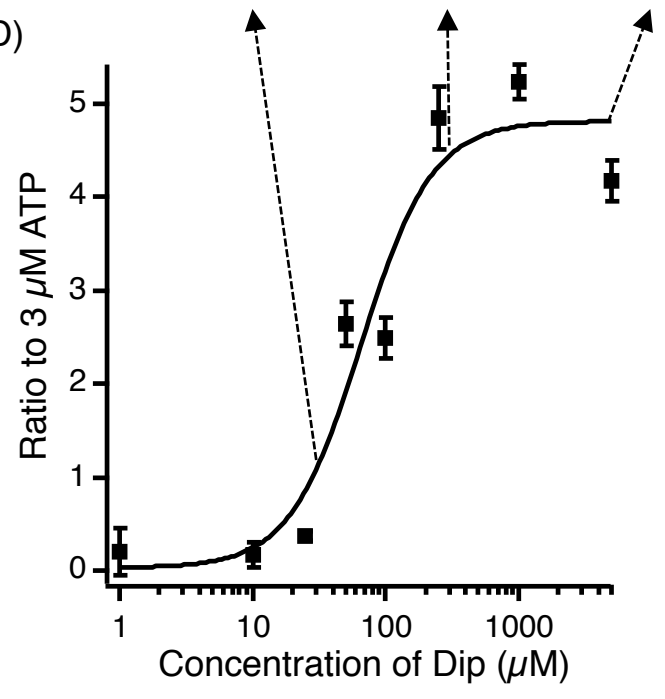

Figure 3

Hirose et al. 